\title{
Sistema de evaluación de la competencia transversal CT-12
}

\section{Cervelló-Royo, Roberto ${ }^{a}$; Estellés-Miguel, Sofía ${ }^{b}$; Ribes-Giner, Gabriela ${ }^{c}$; Ubeda- García, Juan Enrique ${ }^{d}$}

aFacultad de Administración y Dirección de Empresas (FADE), DECS, Universitat Politècnica de València, Valencia (España), rocerro@esp.upv.es; ${ }^{\mathrm{b}}$ Facultad de Administración y Dirección de Empresas (FADE), DOE, Universitat Politècnica de València, Valencia (España), soesmi@omp.upv.es; 'Facultad de Administración y Dirección de Empresas (FADE), DOE, Universitat Politècnica de València, Valencia (España), gabrigi@omp.upv.es; dFacultad de Administración y Dirección de Empresas (FADE), DECS, Universitat Politècnica de València, Valencia (España), juaubgar@esp.upv.es

\begin{abstract}
The European Higher Education System has moved from a learning content to an orientation based on learning results and development of competences. The Faculty of Business Administration and Management (FADE) develops the 13 skils related with the learning of contents. In this communication, we will develop the skill "TS-12 Time planning and management" which was chosen as checkpoint in the following subjects: at $1^{\text {st }}$ level in the "Production and Operation management" and "Financial Mathematics" subjects and at $2^{\text {nd }}$ level in the "Banking and Stock Change", "Human Resources Management" and "Company Valuation". We will explain the evaluation methodologies applied in each one of the subjects and we will also develop the observed advantages and disadvantages in the development of the TS-12 competence. Under the methodological vision of action-research and the critical observation of teaching in the "Production and Operation Management", "Financial Mathematics", "Banking and Stock Change", "Human Resources Management" and "Company Valuation" subjects. We use the theory classes/lectures, the lab-cases/lab-sessions and the exercises portfolio of the students in order to check how the TS-12 is developed.
\end{abstract}

Keywords: Competences, Time planning and management-TS12, Production and Operation management, Financial Mathematics, Banking and Stock Change, Human Resources Management and Company Valuation.

\section{Resumen}

El sistema europeo de educación superior ha pasado de un contenido de aprendizaje a una orientación basada en los resultados de aprendizaje y el desarrollo de competencias. La Facultad de Administración y Dirección de Empresas (FADE) desarrolla las 13 competencias relacionadas con el aprendizaje de los contenidos. En esta comunicación, desarrollaremos la competencia "CT-12 Planificación y Gestión del Tiempo" que fue elegida como punto de control en las siguientes asignaturas: en un $1^{\text {er }}$ nivel en las asignaturas "Dirección de producción y operaciones" y "Matemáticas financieras" y en un $2^{\circ}$ nivel en las asignaturas "Banca y Bolsa", "Dirección 
de Recursos Humanos" y "Valoración de Empresas". Explicaremos las metodologías de evaluación aplicadas en cada una de las asignaturas, asi como el desarrollo de las ventajas y desventajas observadas en el desarrollo de la competencia TC-12. Bajo la visión metodológica de accióninvestigación y la observación crítica de la enseñanza en las asignaturas "Dirección de producción y Operaciones", "Matemáticas Financieras", "Banca y Bolsa", "Dirección de Recursos Humanos" y "Valoración de Empresas". Utilizamos las clases teóricas/seminarios, las prácticas y sesiones de laboratorio y el portafolio de ejercicios de los estudiantes con el fin de comprobar cómo se desarrolla la CT-12

Palabras clave: Competencias, Planificación y Gestión del Tiempo-CT12, Dirección de Producción y Operaciones, Matemáticas Financieras, Banca y Bolsa, Dirección de Recursos Humanos y Valoración de Empresas. 


\section{Introducción}

La constitución del Espacio Europeo de Educación Superior (EEES), supuso una nueva organización de las enseñanzas universitarias en sus diferentes niveles. Conllevando la elaboración de nuevos Planes de Estudio, como el del Grado de Administración y Dirección de Empresas, en la Universidad Politécnica de Valencia (UPV).

La formación en este Grado, incluye, en los primeros cursos, contenidos de aprendizaje básicos, con carácter obligatorio, necesarios para la adquisición de conocimientos relativos a la Dirección y organización de las empresas, como es el caso de Dirección de Operaciones y Producción, Dirección de Recursos Humanos, Matemáticas financieras, Banca y Bolsa y la Valoración de Empresas.

Por otro lado, el EEES trajo consigo un cambio en la orientación de la enseñanza, marcando un nuevo paradigma de aprendizaje en la educación superior pasando de un aprendizaje basaso meramente en contenidos, a una orientación completamente diferente basada en resultados de aprendizaje y desarrollo de competencias (Sanabria-Codesal, y otros 2014).

De esta forma, la universidad actual, cada vez más centrada en la atención al estudiante como persona que se desarrolla en el proceso de aprendizaje profesional. La formación humanística de profesionales competentes y comprometidos con el desarrollo social constituye una preocupación y un objetivo a lograr (Devece Carañana et al, 2011).

El EEES ha creado una serie de propuestas con el objetivo de mejorar la calidad de la educación universitaria, siendo una de ellas la focalización de los procesos de enseñanzaaprendizaje en torno a competencias en un intento de aunar la formación académica (aprendizaje de conocimientos) y el desarrollo personal (Colás, 2005). Las competencias deben ser comportamientos habituales, no esporádicos. Son precisamente los hábitos los que dan a las competencias su carácter predictivo (Ribes et al. 2018).

Algunas de las definiciones sobre el término "competencia” son las siguientes:

- "El buen desempeño en contextos diversos y auténticos basados en la integración y activación de conocimientos, normas, técnicas, procedimientos, habilidades y destrezas, actitudes y valores" (Villa y Poblete, 2007).

- "Como la capacidad de los sujetos para seleccionar acciones ajustadas a las demandas y fines deseados" (Colás, 2005).

- El término "competencias" representa una combinación dinámica de atributos con respecto al conocimiento y su aplicación, a las actitudes y a las responsabilidades-, que describen el nivel o grado de suficiencia con que una persona es capaz de desempeñarlos. Este concepto está estrechamente relacionado con otros términos de significado similar como capacidad, habilidad o destreza. En el informe final del proyecto Tuning, las competencias han sido entendidas como: "conocer y comprender" (conocimiento teórico de un campo académico), "saber cómo actuar" (la aplicación práctica y operativa del conocimiento en 
situaciones determinadas) y "saber cómo ser" (los valores como parte integrante de la forma de percibir a los otros y vivir en un contexto social) (Tunning, 2008).

En la actualidad, la UPV se encuentra implantando un modelo de formación en competencias transversales consideradas necesarias para el desempeño profesional de los titulados de la UPV, al margen de las competencias técnicas. Este modelo se incorporará en el diseño de las propuestas educativas. Entre las competencias transversales planteadas, se plantea la Competencia Transversal 12: "Planificación y Gestión del Tiempo", definida por la UPV (2018) como planificar adecuadamente el tiempo disponible y programar las actividades necesarias para alcanzar los objetivos, tanto académico-profesionales como personales. Hay varios trabajos realizados sobre esta competencia en el entorno universitario (Uve et al., 2016; Montañes et al. 2017; Capó et al, 2018).

La identificación de un problema específico de aprendizaje o de un dilema docente, debe activar procesos de investigación en el aula para examinar y mejorar el aprendizaje de los alumnos (Morales 2012). El fin último será adaptar la docencia a las circunstancias de aprendizaje, buscando el mejor resultado posible en dicho proceso de aprendizaje.

Las asignaturas de "Dirección de producción y operaciones", "Matemáticas financieras", "Banca y Bolsa", "Dirección de Recursos Humanos" y "Valoración de Empresas", incluyen entre las competencias relativas al aprendizaje de los contenidos de la asignatura, la Competencia Transversal-12: Planificación y Gestión del Tiempo, a un primer nivel, las dos primeras y a un segundo nivel, las tres últimas.

Las sesiones de prácticas són un entorno idóneo para el desarrollo y valoración de algunas de estas competencias. Las prácticas informáticas se establecen expresamente en cuanto a duración y contenido en relación a los desarrollos teóricos de las asignaturas, suele disponerse de unas 7 sesiones de prácticas informáticas. Los grupos de prácticas, son numerosos y los alumnos no disponen de un ordenador para cada uno de ellos, obligando a los alumnos a compartir los recursos informáticos del aula por parejas. Por otra parte, los problemas que se plantean en las sesiones de prácticas van encaminados al cumplimiento de la competencia 12 . No pudiendo limitarse a la simple resolución de ejercicios prácticos en ordenador. Siendo necesario un buen aprovechamiento del tiempo en el desarrollo de las sesiones para poder abordar problemas más avanzados con el tiempo suficiente y la planificación adecuada. Por otra parte, resulta de suma importancia contar con información relativa al grado de adquisición por el alumno, de los conocimientos que se pretenden.

\section{Objetivo}

El objetivo de la presente comunicación es presentar una propuesta de evaluación y control para la Competencia Transversal 12: Planificación y Gestión del Tiempo que contribuya a la mejora e integración de dicha competencia en el alumnado en las 
asignaturas de "Dirección de producción y operaciones", "Matemáticas financieras", "Banca y Bolsa", "Dirección de Recursos Humanos" y "Valoración de Empresas".

\section{Desarrollo de la innovación}

La UPV (2018) propone para el desarrollo de esta competencia el desarrollo de las siguientes actividades formativas:

- Actividades grupales

- Estudio de casos

- Contrato de aprendizaje

- Exposiciones orales

- Foros y debates

- Portafolio

- Prácticas de laboratorio

- Problemas

- Proyectos

A modo de ejemplo y en el caso las sesiones de prácticas de laboratorio, los alumnos abordan la resolución problemas reales, a un nivel de dificultad establecido en la asignatura según sus contenidos y estableciendo un control del tiempo (Cervelló-Royo y Marqués Pérez, 2016).

De esta forma., bajo la visión metodológica de action research (investigación-acción), consistente en una orientación metodológica de investigación para mejorar los procesos de enseñanza-aprendizaje, se tratará de dar un enfoque en el que a partir de una reflexión crítica sobre la situación actual, se suceden de manera cíclica fases de puesta en práctica de algún tipo de innovación y de reflexión sobre los resultados, que pueden llevar a una solución práctica, en la docencia de esta asignatura. Por lo tanto y ante la observación crítica de la situación de la docencia de "Dirección de producción y operaciones", "Matemáticas financieras", "Banca y Bolsa", "Dirección de Recursos Humanos" y "Valoración de Empresas" se utilizan las prácticas de laboratorio para para plantar verdaderos problemas y no limitarse a la mera resolución de ejercicios prácticos. Como se ha comentado anteriormente, se trabajará en grupos numerosos que han de compartir los recursos informáticos del aula de prácticas, con la necesidad de gestionar adecudamente el tiempo de las sesiones para alcanzar los objetivos de aprendizaje propuestos. Con todo esto, se plantea un proyecto de innovación y evaluación docente que, resolviendo los problemas de docencia, incorpore la competencia CT-12 "Planificación y Gestión del Tiempo" que favorezca un mejor proceso de aprendizaje.

\subsection{Planificación y Gestión del Tiempo}

La competencia CT-12 "Planificación y Gestión del Tiempo" se implementa a través de las prácticas, ejercicios y pruebas de evaluación previstas.

1. Control de fechas de entrega y deadlines frente a calidad del trabajo. Mediante el control del portafolio que puede adoptar diferentes modalidades para analizar el trabajo 
desarrollado por el alumnado a lo largo de las jornadas de trabajo establecidas para alcanzar unos resultados.

2. Prácticas de laboratorio, en sus tres modalidades:

2.1. Individual autoorganizado: cada uno se organiza su tiempo para responder, pudiendo observarse como cada estudiante planifica el tiempo, como lo emplea y en que lo emplea.

2.2. Individual sobre tiempo de respuesta definido: el estudiante debe responder a las preguntas sobre unos textos y unos datos que se le proporcionan, valorándose el tiempo dedicado a su resolución en relación con el parámetro temporal definido para la consecución del objetivo.

2.3. Grupo autoorganizado, dentro del grupo se reparten las tareas, observándose cómo cada grupo de estudiantes asigna sus tareas y las desarrolla.

3. Establecer holguras de tiempo y ver si lo cumplen, se excede o queda por debajo.

En la Tabla 1 se pueden observar los objetivos y resultados de aprendizaje plantedos para la CT-12 (entre otras) por La UPV (2018)

\section{Objetivos:}

Desarrollar en los alumnos una actitud mental mediante la aplicación de procedimientos estructurados de resolución de problemas que promueva su capacidad de aprender, comprender y aplicar conocimientos de forma autónoma

Contribuir al desarrollo de otras competencias básicas como el trabajo en equipo, la creatividad, el análisis o el liderazgo

Favorecer que el alumno incorpore en la resolución de los problemas las tareas previas de identificación, y de programación y planiciación de las actuaciones a desarrollar para la resolución de los mismos

Familiarizar al alumno en las tecnologías más avanzadas en el ámbito profesional para el que se forma

\section{Resultados de aprendizaje:}

Identificar y analizar un problema para generar alternativas de solución, aplicando los métodos aprendidos: definir con exactitud el problema a resolver, recopilar la información significativa para la solución

Utilizar la experiencia y el criterio para analizar las causas de un problema y construir una solución más eficiente y eficaz: identificar bien las causas que generan el problema, identificar las posibles alternativas para la solución, evaluar ventajas y desventajas de cada alternativa, decidir la solución oportuna, decidir la aplicación y el seguimiento de la misma 
Definir claramente los objetivos a cumplir, establecer un orden de prioridades para la resolución de los mismos, y programar y planificar las actividades para la resolución

Aplicar las herramientas propias del ámbito profesional. En concreto la Hoja de cálculo, y las funciones financieras que incorpora.

Fuente: Documento UPV “Competencias Transversales”.

\subsection{Gestión del Tiempo}

Por otra parte, la resolución de casos y supuestos se realiza con fecha límite de entrega que normalmente suele ser la propia sesión de prácticas. La valoración de la eficiencia en la Gestión del Tiempo (CT-12) para la resolución de los mismos, se realiza comprobando el desarrollo de la práctica y la entrega de resultados, incorporando en algunas asignaturas una valoración por grupos del tiempo de entrega de resultados respecto de la estimación de tiempo óptimo que debe emplearse en la resolución..

El desarrollo de cuestionarios a través de PoliformaT aporta datos sobre el día y la hora de entrega de la práctica, así como el tiempo empleado por cada alumno, permitiendo analizar detalladamente el tiempo empleado por cada alumno en la resolución, y pudiendo establecer de esta forma comparaciones con el conjunto de los alumnos.

\subsection{Ventajas e inconvenientes observados}

\section{Ventajas}

- Que aprendan a gestionar su tiempo. Individualmente y por equipo.

- Distribuirse las tareas para alcanzar los objetivos en un plazo determinado de tiempo.

\section{Inconvenientes}

- Dificultad en la Gestión del tiempo cuando se trabaja en grupo.

- Planificación y reparto de las tareas.

- Problema de postergar el desarrollo de la tarea, dejándolo todo para el final.

\section{Conclusiones}

Entre las competencias transversales trabajadas en el Grado de Administración y Dirección de Empresas de la Universidad Politécnica de Valencia, se trabaja como punto de control la CT-12. Planificación y Gestión del Tiempo. Bajo la visión metodológica de action research (investigación-acción), y ante la observación crítica de la situación de la docencia de "Dirección de producción y operaciones", "Matemáticas financieras", "Banca y Bolsa", "Dirección de Recursos Humanos" y "Valoración de Empresas" se utilizaron las prácticas en laboratorio, los ejercicios y las pruebas de evaluación para plantear casos y problemas reales con la necesidad de que el alumno deba gestionar adecudamente el tiempo de las sesiones para alcanzar los objetivos de aprendizaje propuestos. De esta 
manera, mediant la observación y la evaluación, se pudo comprobar como con la resolución de dichos problemas de docencia, se consigue incorporar la competencia transversal CT-12 "Planificación y Gestión del Tiempo" en el desarrollo de las tareas a nivel individual, grupal y a lo largo del curso. Los resultados obtenidos demuestran que con la introducción de casos reales en el portafolio, ejercicios y en las prácticas de laboratorio así como el uso de las TIC y las herramientas ofimáticas bajo el prisma del action-research, se consigue controlar y evaluar dicha competencia.

\section{Referencias}

CAPÓ VICEDO, J., MULA, J., DÍAZ-MADROÑERO BOLUDA, F. M., \& VICEDO PAYÀ, P. (2018). Aplicación de una metodología de trabajo ágil y colaborativo (SCRUM) en el Master Universitario en Dirección de Empresas (MBA) para la mejora de las competencias transversales.

CERVELlÓ ROYO, R.; MARQUÉS PÉREZ, I. (2016). Mejora del Sistema de evaluación de las prácticas con ordenador en la asignatura de Matemáticas Financieras en el Grado de ADE incorporando las competencias transversales CT-3, CT-6, CT-12 y CT13. EN Congreso Nacional de Innovación Educativa y Docencia en Red = Congrés Nacional d'Innovació Educativa i Docència en Xarxa (IN-RED 2016). (1 - 16). Valencia, España: Editorial UPV.

COLAS, P. (2005). La universidad en la Unión Europea. Ed. Alianza, Málaga, pp. 104107.

DEVECE CARAÑANA, C.A.; GRAU GADEA, G.F., BARBERÁ RIBERA, M.T. Y ESTELLES-MIGUEL, S. (2011). Collaborative methodologies to obtain specific competences on information management in public administration studies, Congreso Edulearn 2011, pp. 5724-5731.

HERNÁNDEZ PINA, F; MARTÍNEZ, P. DA FONSECA Y RUBIO, M. (2005). Aprendizaje, competencias y rendimiento en educación superior. Ed. La Muralla, pp. 52.

MONTAÑÉS MUÑOZ, N., BALART, R., SÁNCHEZ NACHER, L., QUILES CARRILLO, L. J., \& FOMBUENA, V. (2017, July). Buenos días: la clase de hoy es un juego $i$ y porqué no?. In In-Red 2017. III Congreso Nacional de innovación educativa y de docencia en red. (pp. 438-445). Editorial Universitat Politècnica de València.

MORALES, P. «Investigación en Innovación Educativa.» Revista Iberoamericana sobre Calidad, Eficacia y Cambio en Educación 8, $\mathrm{n}^{\circ} 2$ (2012).

RIBES GINER, G.; PERELLÓ MARÍN, MR.; HERRERO BLASCO, A. (2018). Dirección de recursos humanos. Dirección de personas. Editorial Universitat Politècnica de València. http://hdl.handle.net/10251/113116

SANABRIA-CODESAL, E., BOSCH, I. VINCENT-VELA, MC., LLORET, J., ÁLVAREZ-BLANCO, S. y ROMERO PÉREZ, L. (2014). «Análisis de las Dimensiones 
Competenciales Incluidas en Diferentes Asignaturas en Ingenierías.» Editado por Universidad Politécnica de Valencia. Jornadas de Innovación Educativa y de Docencia en Red. Valencia: Universidad Politécnica de Valencia. 1050-1064.

Tunning (2008). Proyecto Tuning Educational Structures in Europe 2008. Disponible en http://tuning.unideusto.org/tuningeu/ (consultado en octubre de 2009)

UPV (2018) Documento Competencias Transversales. www.upv.es/contenidos/COMPTRAN/info/955144normalc.html

UVE, G. E. C., SORIA, E. E. A., SUQUILANDA, C. F. R., \& PÉREZ, M. J. R. (2016). Proyectos integradores de saberes como estrategia didáctica de aprendizaje en los estudiantes del Instituto Tecnológico Superior Julio Moreno Espinosa. Cuadernos de Educación y Desarrollo, (69).

VILLA, A. y POBLETE, M. (2007). Aprendizaje basado en competencias. Una propuesta para la evaluación de competencias genéricas. Vicerrectorado de Innovación y Calidad Universidad de Deusto, Ed. Mensajero, Bilbao, pp. 24-25. 